\title{
Trägergranula und Ommochrompigmente in den Augen verschiedener Rassen und Transplantat-Träger . von Ephestia kühniella Z. und Ptychopoda seriata Schrk.
}

\author{
Von Gisela Hanser
}

\author{
Aus dem Kaiser-Wilhelm-Institut für Biologie, Abt. K üh n, Hechingen
}

(Z. Naturforschg. 1, 396-399 [1946]; eingegangen am 26. April 1946)

Die Ommochromfarbstoffe der Augenpigmente von Ephestia und Ptychopoda sind an Eiweiß-Trägergranula gebunden. Das Corneazellpigment unterscheidet sich von dem der übrigen pigmentierten Ommatidienzellen in der Pigmentkorngröße; an Stelle von Skotommin führt es in den Ephestia-Augen Xanthommin. Durch $a$ wird der Skotommin- und der Xanthommingehalt vermindert, durch $a+$-Stoff-Zufuhr (durch +-Implantate oder Kynurenin-Injektion) wird er in $a$-Tieren gesteigert. In den Augen der wa-Ephestien fällt die zur Pigmentbildung notwendige Trägersubstanz aus. Das hellgelbe Pigment der dec-Ptychopoda-Augen färbt sich im kurzwelligen Licht rot aus. Die Fähigkeit zur +-Pigmentbildung in $a$-Augen nach +-Stoff-Zufuhr beginnt und erlischt in den einzelnen Zellelementen zu verschiedenen Zeiten.

$\mathrm{D}$ ie genabhängige Augenpigmentbildung bei Insekten bietet ein günstiges Modell entwicklungsphysiologisch-genetischer Vorgänge ${ }^{1}$.

Aus Tryptophan wird unter Wirkung eines Gens $\left(a^{+}\right.$-Ephestia $\left.=v^{+-D r o s o p h i l a}\right)$ Kynurenin gebildet $^{2}$ und aus diesem das Ommochrom, der Farbstoff der Insekten-Augenpigmente, aufgebaut ${ }^{3,4}$. Die Untersuchung der quantitativen Beziehung zwischen zugeführtem Kynurenin und Ommochrom in den Ephestia-Augen ergab, daß etwa doppelt soviel Skotommin (Ommochrom der Schmetterlingsaugen) gebildet wie Kynurenin zugeführt wird $^{5}$, also entsteht das Skotommin aus Kynurenin in Verbindung mit andern, noch unbekannten Stoffen. In den Dipterenaugen bildet sich aus Kynurenin Ommatin, ein Ommochrom von geringerer Molekulargröße.

Die fertigen Augenpigmente sind im Pigmentkorn mit Eiweiß zu einem Chromoproteid verbun$\operatorname{den}^{4}$. Die letzte Stufe der Pigmentbildung verläuft strukturgebunden auf einer Trägersubstan $z^{6}$, die wahrscheinlich mit dem Eiweißkörper des Pigmentkorns identisch ist. Diese strukturgebundenen Vor-

1 Zusammenfassung: A. K ühn, Nachr. Ges. Wiss. Göttingen, Math.-Physik. Kl. [1941].

2 A. Butenandt, W. Weid el u. E. B ecker, Naturwiss. 28, 63 [1940].

3 E. B ecke r, Biol. Zbl. 59, 597 [1939].

4 E. B e c k e r, Z. indukt. Abstammg. Vererbungsl. 80, 157 [1942]. gänge werden, wie Transplantationsversuche ${ }^{7,8}$ zeigten, durch die Mutation wa bei Ephestia und dec bei Ptychopoda, ebenso wie durch $w$ bei Drosophila ${ }^{9}$, gestört.

Außer dem in Ammoniak und Ameisensäure löslichen Skotommin (der „Rotkomponente“) läßt sich noch eine wasserlösliche "Gelbkomponente" aus den Ephestia-Augen extrahieren. Letztere zerfällt in undialysables Xanthommin und einen dialysablen Anteil ${ }^{3}$.

Die Zellelemente eines Ommatidiums des Superpositionsauges von Ephestia und Ptychopoda zeigt Abb. 1. Das Pigment häuft sich hauptsächlich in den Nebenpigmentzellen; die Retinulazellen sind bei Ephestia distal der Kernzone pigmentiert, bei Ptychopoda pigmentfrei. Die Corneapigmentzellen enthalten eine einschichtige Pigmentkornlage.

Bei +-Ephestia stellte ich zwei verschiedene Augenpigmente in Granulaform fest: ein dunkelbraunes, in Wasser unlösliches Pigment in den Retinula- und Nebenpigmentzellen und ein dunkelgelbes, wasserlösliches Pigment in den Corneapigmentzellen, dessen Pigmentkörner sich auch

5 A. K ühn u. E. B e ck e r, Biol. Zbl. 62, 303 [1942].

6 R. D a n n e e l, Biol. Zbl. 61, 388 [1941].

7 A. Kühn u. V. Schwartz, Biol. Zbl. 62, 226 [1942].

8 V.Schwartz, Biol. Zbl. 61, 253 [1941].

9 B. Ephrussi u. S. Chevais, Bull. Biol. France Belg. 72 [1938]. 


\begin{tabular}{|c|c|c|c|c|c|c|c|c|c|c|}
\hline & \multicolumn{6}{|c|}{ Ephestia } & \multicolumn{4}{|c|}{ Ptychopoda } \\
\hline & \multicolumn{2}{|c|}{+} & \multicolumn{2}{|c|}{$a$} & \multicolumn{2}{|c|}{ wa } & \multicolumn{2}{|c|}{+} & \multicolumn{2}{|c|}{$d e c$} \\
\hline & Pigment & $\begin{array}{l}\text { entpig- } \\
\text { mentiert }\end{array}$ & Pigment & $\begin{array}{l}\text { entpig- } \\
\text { mentiert }\end{array}$ & Pigment & $\begin{array}{l}\text { entpig- } \\
\text { mentient }\end{array}$ & Pigment & $\begin{array}{l}\text { entpig- } \\
\text { mentient }\end{array}$ & Pigment & $\begin{array}{l}\text { entpig- } \\
\text { mentient }\end{array}$ \\
\hline $\begin{array}{c}\text { Comea- } \\
\text { pigmentzellen }\end{array}$ & & $0 y$ & & 0 & - & - & & 0 & ? $_{L}$ & 0 \\
\hline $\begin{array}{c}\text { Neben- } \\
\text { pigmentzellen }\end{array}$ & $\mathcal{S}$ & $\circ$ & ${ }^{\circ}{ }_{S}$ & 0 & - & - & $s$ & $\circ$ & ${ }^{3}\left(\begin{array}{l}L \\
\end{array}\right.$ & 0 \\
\hline Retinulazellen & $\mathcal{S}$ & ○ & (7) & ○ & - & - & - & - & - & - \\
\hline
\end{tabular}

Tab. 1. Schematischer Vergleich der Pigmentarten, Pig mentmengen und Granulagrößen in verschiedenen Zellelementen bei verschiedenen Rassen von Ephestia küh niella und Ptychopoda seriata. $X=$ Xanthommin, $S=$ Skotommin, $L=$ im Licht aus färbbares Pigment.

durch größere Dimensionen von dem der übrigen Zellen unterscheidet (Abb. 1, Tab. 1). Das dunkelbraune Pigment zeigt das für Skotommine charakteristische Redoxverhalten ${ }^{4}$. Bei dem dunkel-

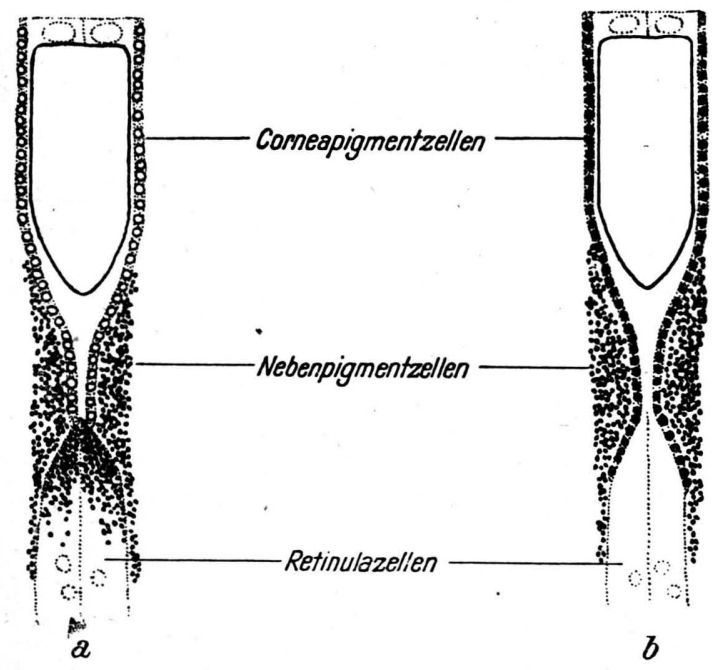

Abb. 1. Distalabschnitte von Ommatidien der Wildformen in Hellstellung (schematisch) a) Ephestia, b) Ptychopoda.

gelben Pigment der Corneapigmentzellen handelt es sich vermutlich um den von B e ck e r mit Xanthommin ${ }^{3}$ bezeichneten Stoff; darauf läßt die Wasserlöslichkeit schließen, das Redoxverhalten fehlt. Mit Heidenhainschem Hämatoxylin gefärbte Schnitte durch ein +-Auge enthalten in den Corneapigmentzellen kein Pigment mehr, sondern nur blaugefärbte Granula. Aus ihrer Verteilung kann man schließen, daß sie die Trägergranula des her-

10 W. Um b a c h, Z. Morph. Ökol. 28, 561 [1934].

11 V. V o in ov, Arch. Zool. exp. gén. 67, 233 [1928]. ausgelösten gelben Farbstoffs sind. Nach Entpigmentieren durch essigsaures $\mathrm{NaNO}_{2}$ oder mittels der Palschen Bleichmethode bleiben auch an Stelle der dunkelbraunen Pigmentkörner der übrigen pigmentierten Zellen mit Hämatoxylin schwach färbbare Granula zurück (Tab.1). Die Trägergranula besitzen eine geringe Größe (ca. $0,2 \mu$ ).

In der +-Puppe der Wildrasse tritt das Pigment zunächst in den Retinulazellen (0-200 Stdn. alte Puppe bei $20^{\circ}$ Entwicklungstemperatur), von einem dorso-caudalen Augenbezirk aus über die Ommatidien fortschreitend, auf ${ }^{10}$. In den Nebenpigmentzellen entsteht das Pigment im Alter von 220 bis 240 Stdn. in allen Ommatidien gleichzeitig. Zuletzt erscheint das gelbe Xanthomminpigment in den Corneapigmentzellen (am 13. Tag der Puppenruhe). Mit zunehmendem Alter des Einzelkorns gehen Wachstum und Farbvertiefung einher. Als Vorstufe der Pigmentkörner sieht man in den Nebenpigmentzellen (220 Stdn.) mit $\mathrm{He}$ i d e $\mathrm{n}$ h a in Hämatoxylin blaß blau gefärbte Granula auftreten, die sich allmählich durch braunes Pigment verdunkeln und wachsen. In den Retinulazellen sind Prägranula durch das dichte Plasma und chromatinreiche Kerne nicht sichtbar. Eine Beziehung der Prägranula zu Mitochondrien, die Voinov ${ }^{11}$ als Pigmentvorstufe bei Simulium-Larven fand, konnte ich nicht feststellen; die $\mathrm{Ku} / 1$ sche Mitochondrienfärbung fiel negativ aus.

$\mathrm{Zu}$ dem Gen $a^{+}$kennen wir die Allele $a$ (',rotäugig") und $a^{\mathrm{k}}$ (,,kaffeebraunäugig“" ${ }^{12}$. Das Augenpigment der $a$-Falter ist kleinkörniger als das von $a^{+13}$, zeigt aber entsprechende Verteilung und Korngrößenunterschiede wie in den Zellen der

12 H. Pi ep h o, Roux' Arch. 133, 495 [1935].

13 E. Plag g e, Roux' Arch. 132, 648 [1935]. 
Schwarzäugigen. Das Pigment der Corneapigmentzellen ist wie im Wildauge grobkörniger als das der übrigen Pigmentzellen (Tab. 1). Der Vergleich ungefärbter Augenschnitte im Vergleichsmikroskop läßt erkennen, daß die Pigmentkörner der Corneapigmentzellen im $a$-Auge etwas heller gefärbt und kleiner als im +-Auge sind. Also wird nicht nur der Skotommingehalt der Retinula $L$ und Nebenzellpigmentkörner, sondern auch der Xanthommingehalt der Granula in den Corneapigmentzellen durch a vermindert. Dieser histologische Befund stimmt mit Beckers ${ }^{3}$ Feststellung bei der chemischen Analyse überein. Die Ommochrome sind im $a$-Auge wie im Wildauge an Trägergranula gebunden (Tab. 1), die nach Entpigmentierung zurückbleiben. In den $a$-Puppen erfolgt die Pigmentbildung in derselben Reihenfolge wie bei den Wildaugen $^{13}$, aber das $a$-Pigment bildet sich ir einem späteren Entwicklungsstadium der Zellen.

Die weißäugige Ephestia-Mutante $w a^{1,8} \mathrm{kann}$ $a^{+}$kryptomer führen; wa wird immer manifest, wenn das mutierte Allel homozygot vorhanden ist. Transplantate mit $w a$ lösen in $a$-Wirtsaugen Ommochrombildung aus; $w a$-Augenimplantate

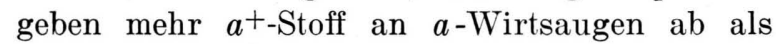
+-Implantate, da $w a$-Augen selbst kein $a^{+}$-Pigment bilden ${ }^{7}$. Die histologische Untersuchung von $w a$ zeigt, daß nicht nur das Pigment fehlt, sondern auch keine mit Hämatoxylin färbbaren Granula gebildet sind (Tab. 1). Das wa+-Gen greift bei der Prägranula-Bildung in den Entwicklungsgang ein; wa unterdrückt die zur Augenpigmentsynthese notwendige Bildung von Trägersubstanz.

Bei Ptychopoda fehlt in den Retinulazellen das Pigment vollständig (Abb.1b). In den Corneapigmentzellen liegen wie bei $a^{+}$größere Pigmentgranula als in den Nebenpigmentzellen; sie führen aber kein Xanthommin wie bei Ephestia, sondern dunkelbraunes Skotommin wie die Nebenpigmentzellen (Abb. 1b, Tab. 1). An Hand der Korngröße des Corneazellpigments kann man im helladaptierten Auge feststellen, daß die Corneapigmentzellen sehr weit proximalwärts reichen und die distale Spitze der Retinulazellen glockenförmig umfassen (Abbildung $1 \mathrm{~b}$ ); sie können auf diese Weise das fehlende Retinulazellpigment bei Ptychopoda in der Hellstellung funktionell ersetzen.

Die Mutation dec von Ptychopoda hat im Dunkeln aufgezogen hellgelbe Augen. Das dec-Pigment ist kleinkörniger als das des dec ${ }^{+}$-Auges und fehlt wie bei der Wildform in den Retinulazellen. Die
Pigmentkörner der Corneapigmentzellen sind gröBer als die der Nebenpigmentzellen, aber ebenfalls hellgelb (Tab.1). Der gelbe Farbstoff des Corneaund Nebenzellpigments löst sich in Wasser. Im Augenpigment-Ausstrich bleiben bei $\mathrm{dec}^{+}$und dec nach Pigmententfernung Trägergranula wie bei $a^{+}$und $a$ zurück (Tab.1). Die dec-Augen besitzen die Eigentümlichkeit, daß sie sich im Licht von Gelb über Orange zu Rot ausfärben ${ }^{14}$. Die Ausfärbung beruht auf direkter Lichtwirkung; bei einseitiger Beleuchtung wird die vom Licht abgewandte Seite nicht ausgefärbt. Zur Ermittlung der bei der Ausfärbung wirksamen Wellenlängen wurden die Köpfe der dec-Falter mit einem QuecksilberMonochromator bestrahlt ${ }^{15}$. Die größte Wirksamkeit zeigten Strahlen der Wellenlänge $313 \mathrm{~m} \mu$, kurzwelligere Strahlen $(302 \mathrm{~m} \mu)$ wirken tödlich. Bei $\lambda$ $=546 \mathrm{~m} \mu$ erfolgt keine Pigmentausfärbung. Das histologische Präparat zeigt, daß die gelben Pigmentgranula in Cornea- und Nebenpigmentzellen sich bei Belichtung in gleicher Weise rot ausfärben und an Größe zunehmen. In welcher chemischen Beziehung das lichtausfärbbare dec-Pigment zum Skotommin steht, kann durch die histologische Untersuchung nicht beantwortet werden. Es könnte ein Zusammenhang zwischen dec-Pigment und dem Stoffbestand bestehen, der neben Kynurenin zur Hälfte an der Skotomminbildung beteiligt ist; oder es könnte sich um einen Stoff außerhalb des bisher bekannten Weges der Augenpigment-Synthese handeln. Daß das gelbe dec-Pigment an denselben Granula entsteht, die in dec ${ }^{+-A u g e n ~ d a s ~}$ Skotommin bilden, wird dadurch bewiesen, daß die dec-Pigmentkörner dieselbe Lage in den Zellen und dieselben Größenunterschiede zwischen Corneazellpigment einerseits und Nebenzellpigment andererseits aufweisen. Transplantationsversuche ${ }^{814}$ zeigen, daß dec-Tiere +-Stoff bilden, den sie selbst nicht zur Skotomminsynthese verwenden können. Ob man bei der Mutation $\mathrm{dec}^{+} \rightarrow$ dec den Grund dafür in einer Änderung der Natur des Trägereiweißes oder des Fermentsystems, welches normalerweise bei der Skotomminbildung beteiligt ist, suchen muß, kann durch die histologische Untersuchung nicht entschieden werden.

Die Retinulazellen der Augen von Ptychopoda und wa-Ephestia machen, obwohl sie kein Pigment

14 A. K ü hn, Naturwiss. 27, 787 [1939]. - A. K ü hn, Z. indukt. Abstammg. Vererbungsl. 78, 1 [1940].

15 Die Versuche wurden im Institut für Strahlenforschung der Universität Berlin durchgeführt; ich danke Hrn. Prof. Dr. Fried r i ch für seine Unterstützung. 
enthalten, bei Dunkeladaptation dieselben Formveränderungen durch wie die Retinulazellen der +-Augen.

Die Pigmentbildung in den einzelnen Zellelementen des Ommatidiums im $a$-Auge nach $a^{+-S t o f f-Z u-~}$ fuhr ist von dem Zeitpunkt der Zufuhr abhängig. Die histologische Untersuchung der durch +Augenimplantate ausgefärbten $a$-Falteraugen läßt drei Stufen unterscheiden:

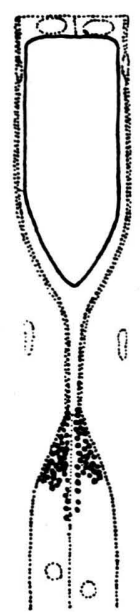

a

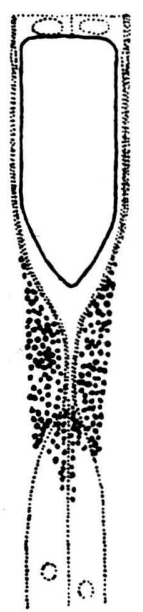

$b$

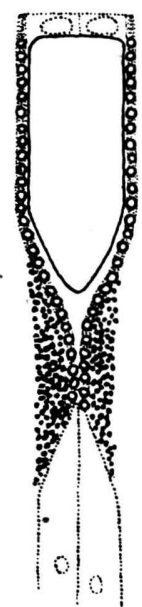

c
Abb. 2. Durch +-Implantate ausgefärbte Augen von a-Ephestia (distale Ommatidien-Abschnitte, schematisch) : a) +-Implantat in Vorpuppe, b) +-Implantat in junge Puppe, c) +-Implantat in ältere Puppe.

1. Wird ein Stück +-Auge in das Auge einer $a$ Vorpuppe eingepflanzt, so bildet sich im Wirtsauge +-Pigment in den Retinulazellen; Neben- und Corneapigmentzellen enthalten nur $a$ entsprechendes Pigment (Abb. 2a).

2. Nach Implantation in eine junge Puppe wird im Wirtsauge in den Retinulazellen nur wenig, in den Nebenpigmentzellen reichlich +-Pigment gebildet; in den Corneapigmentzellen ist das $a$ Pigment nicht ausgefärbt (Abb.2b).

3. +-Implantate in ältere Puppen veranlassen im Wirtsauge in Neben- und Corneapigmentzellen
+-Pigmentbildung. Die Retinulazellen bleiben ohne +-Pigment (Abb. 2c).

Injiziert man $10 \gamma$ Kynurenin in eine $1-5$ Tage alte $a$-Puppe, so entspricht die Pigmentierung der Imaginalaugen der zweiten Stufe, nur sind auch die Corneapigmentzellen +-gemäß ausgefärbt, da der +-Stoff, im Gegensatz zu dem von Implantaten gelieferten, im Überschuß zur Verfügung steht. Bei Kynurenin-Injektion nach mehr als 7 Tagen Puppenruhe entspricht die Pigmentbildung der Stufe 3. Nach dem 12. Tag nach der Verpuppung bleiben +-Implantate ${ }^{16}$ und Kynureningaben ${ }^{5}$ wirkungslos; die Zellen haben ihr Ausfärbungsvermögen verloren. Die einzelnen Zellelemente des Auges sprechen also zu verschiedenen Entwicklungszeiten auf +-Stoff in unterschiedlichem Maße an.

Nach Implantation von Augenanlagen (0 bis 24 Stdn. nach Verpuppung) von Ptychop'oda dec ${ }^{+}$ und dec in Ephestia a stellte sich heraus, daß die $a$-Falteraugen mit $d e c^{+-I m p l a n t a t e n ~(9 ~ E x e m-~}$ plare) die Pigmentbildung entsprechend Abb. $2 \mathrm{a}$, die mit dec-Implantaten (4 Exemplare) die Pigmentierung entsprechend Abb. $2 \mathrm{c}$ aufweisen. Die Pigmentbildung entsprechend Abb. $2 \mathrm{~b}$ tritt bei eec $^{+-}$ (3 Exemplare) und dec-(4 Exemplare) Transplantatträgern auf. Offenbar setzt die +-Stoffabgabe bei dec später ein als bei dec ${ }^{+}$.

Die Transplantations- und Injektionsversuche zeigen außerdem, daß nicht nur die Skotommin-, sondern auch die Xanthomminsynthese in den Corneapigmentzellen durch + -Stoff-Zufuhr gesteigert wird. Hiermit wird die Verwandtschaft des Xanthommins mit den Ommochromen, die B e c k e ${ }^{3}$ vermutete, bewiesen. Hierauf wies auch die Tatsache hin, daß bei Ptychopoda die den XanthominGranula von Ephestia entsprechenden großen Granula der Corneapigmentzellen Skotommin bilden.

In den Ephestia-Augen sind die Zellelemente des Ommatidiums nicht nur morphologisch, sondern auch in ihrem chemischen Bildungsvermögen differenziert.

${ }_{16}$ E. Plagge, Z. indukt. Abstammg. Vererbungsl. 72, 127 [1936]. 